\title{
Heliostat Field Cleaning Scheduling for Solar Power Tower Plants: A Heuristic Approach
}

\author{
Thomas Ashley *, IMUS - Instituto de Matemáticas de la Universidad de Sevilla, Spain. tashley@us.es \\ Emilio Carrizosa, IMUS - Instituto de Matemáticas de la Universidad de Sevilla, Spain. ecarrizosa@us.es \\ Enrique Fernández-Cara, Dep. EDAN and IMUS, Universidad de Sevilla, Spain. cara@us.es
}

October 31, 2018

\begin{abstract}
Soiling of heliostat surfaces due to local climate has a direct impact on their optical efficiency and therefore a direct impact on the productivity of the Solar Power Tower plant. Cleaning techniques applied are dependent on plant construction and current schedules are normally developed considering heliostat layout patterns, providing sub-optimal results. In this paper, a method to optimise cleaning schedules is developed, with the objective of maximising energy generated by the plant. First, an algorithm finds a cleaning schedule by solving an integer program, which is then used as a starting solution in an exchange heuristic. Since the optimisation problems are of large size, a p-median type heuristic is performed to reduce the problem dimensionality by clustering heliostats into groups to be cleaned in the same period.
\end{abstract}

Keywords: Solar Energy; Routing Problems; Scheduling; Cluster Analysis

\section{Introduction}

Research into renewable energy sources has continued to increase in recent years. This is the case, in particular, for the research and application of solar energy systems [10]. Concentrated Solar Power (CSP) is a method of solar energy collection, where the energy from the Sun is concentrated by a field of heliostats onto a central receiver.

A CSP plant can contain a field with thousands of heliostats, where each mirror requires physical access to perform maintenance and cleaning duties. When designing a

\footnotetext{
${ }^{*}$ Corresponding Author

Instituto de Matemáticas de la Universidad de Sevilla (IMUS)

Edificio Celestino Mutis - 1 Planta

Avda. Reina Mercedes, s/n, 41012 Sevilla, Spain.

Tel: +34 955420870.

Email address: tashley@us.es
} 
solar plant, accessibility to each heliostat is an important factor when locating them in the field, which must be optimised in terms of power generation potential [9].

Optimising the subsystems of a solar power plant, such as heliostat aiming strategies [2, 29], multiple receivers [8] and heliostat design [26], are of great interest in the literature.

Accessibility is a driving factor in the use of standard structured heliostat field designs, such as the radially staggered pattern [22]. Structured designs allow easy access to all heliostats for maintenance, and also partially pre-determine the way in which the heliostat field will be cleaned by their structure of rows of heliostats. On the other hand, utilising a pattern-free field design is shown to increase the efficiency of the heliostat field $[7,11]$, but complicates the accessibility of heliostats within the field for both maintenance and cleaning.

Heliostats are required to be cleaned regularly, as accumulation of dust and foreign debris will lower the reflectivity of the mirror and therefore lower the efficiency of the solar plant $[21,24]$.

Various strategies have been developed for cleaning heliostat fields [13]. However, the most widely implemented method is the use of a vehicle with a cleaning arm, which cleans heliostats with a mixture of water and brushing. Cleaning all heliostats daily is impractical due to the number within the field and water scarcity is common in regions with high solar radiation. The frequency of cleaning for each heliostat is partially determined by its physical location within the field, as the energy generated by the heliostat is strongly dependent on its location, see Figure 1 in [9], and heliostats that provide more energy are of more importance to be kept clean. Moreover, the proximity between heliostats and to structures will cause shielding from wind, which can affect dust deposition [25].

The vehicle used for the cleaning activities in a CSP plant has a limited water carrying capacity, and will need to return to the water depot once empty. This limited water capacity, and length of time taken to clean each individual heliostat, will determine a maximum number of heliostats able to be cleaned in a certain cleaning period. Therefore in order to clean an entire field of heliostats, a cleaning schedule is desired, where the objective is to maximise the overall amount of incident energy reflected onto the receiver, under constraints on the number of heliostats cleaned per time period.

The goal of this study is to optimise the cleaning schedule for CSP plants with any size and shape heliostat field. To do this, we will consider the optical efficiency of the heliostats in the field, and also the subsequent routing problems for each period in the schedule.

Whilst this study is applicable to heliostat fields in any general solar power plant, the case of most interest is in a Solar Power Tower (SPT) plant, where the receiver is mounted on top of a tower. In this type of solar plant, the heliostat field layout generally has a more complex geometry, causing the optimal scheduling of cleaning activities to be complicated.

In SPT plants, the concentrated incident radiation and the resultant thermal load is used to drive a steam generator. The SPT plant is usually formed of at least one 
central tower, with a field of heliostats that are able to rotate, in order to track the movement of the Sun and focus the incident radiation onto the receiver surface, allowing high temperatures to be achieved.

SPT plant design is an increasingly investigated research area into renewable energy production. See [4] for a review of heliostat field layout algorithms and see [6] for a computationally efficient method for the design of a heliostat field. A projection method for solar flux distribution is developed in [23] and a novel simulation approach to model radiation transfer is given in [28].

The global cleaning strategy combines an allocation problem, whereby heliostats are allocated to particular cleaning periods, with a routing problem for each period. These problems are not independent, as the route taken by a cleaning vehicle in a particular period will affect the heliostats allocated across the cleaning schedule.

The main innovative contribution of this paper, summarised in the pseudocode in Figure 1, is precisely the way this complex scheduling-routing problem is addressed: we first perform a clustering analysis to divide the field into homogeneous groups, then we determine the optimal cleaning schedule, and finally a local search is performed to improve this sequential solution.

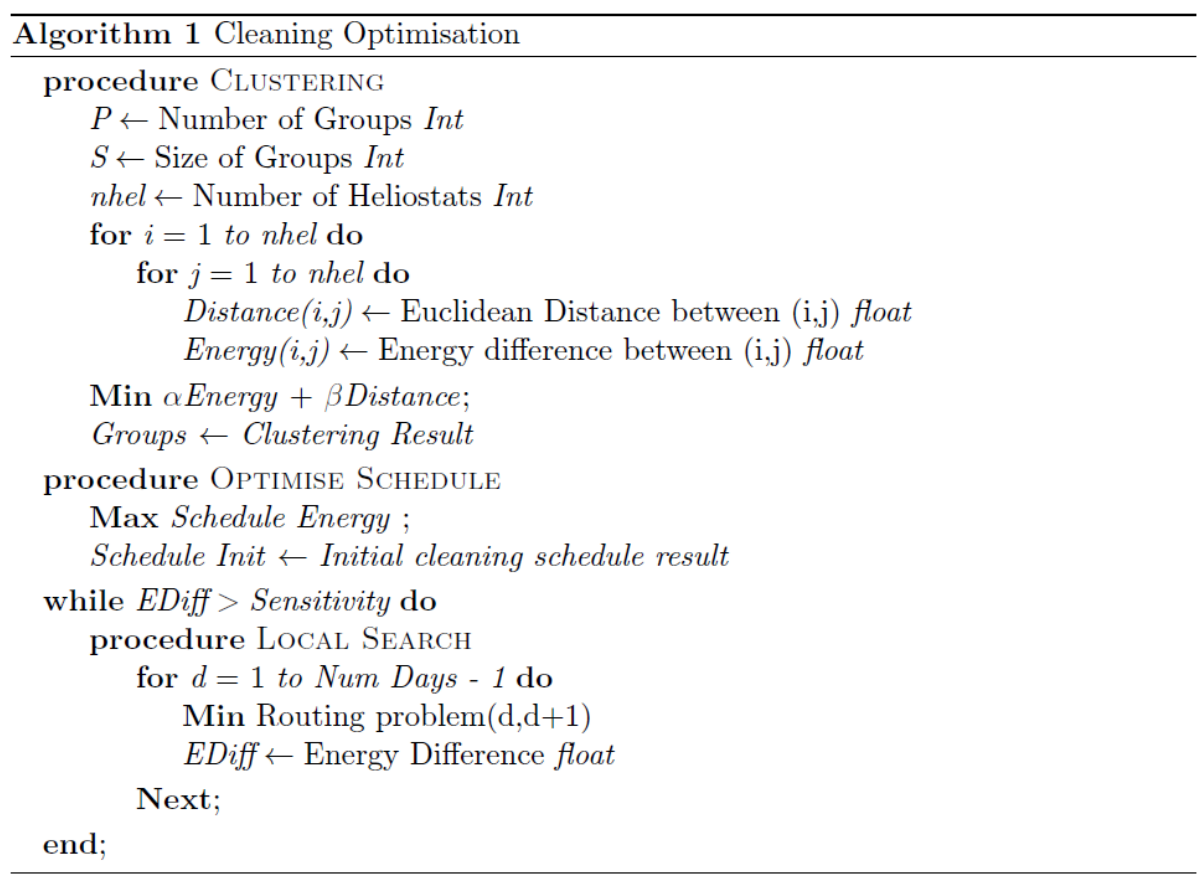

Figure 1: Pseudocode

The structure of this paper is as follows. In Section 2, energy transmission through the system is modelled and degradation in optical efficiency due to soiling is investigated; A grouping optimisation problem is presented, followed by an optimisation procedure to find the cleaning schedule. A subsequent local search heuristic is then applied to 
improve the so obtained solution. Section 3 illustrates our approach with results. Finally, conclusions are given in Section 4.

\section{Modelling}

In this section, the modelling of energy within the SPT system is derived, and the effect of heliostat soiling on efficiency of energy transfer is detailed. The incident solar radiation reflected by each heliostat $h$ at any given time instant $t$, labelled $E_{h t}$, is calculated and stored in a preprocessing step. The method used to model the movement of incident radiation within the system is detailed in [2].

\subsection{Efficiency Degradation}

If heliostat soiling is not considered, the energy reflected by heliostat $h$ at time $t$ is $E_{h t}$ and thus the energy generated in a period of length $T,[0, T]$ is $\int_{0}^{T} E_{h t} d t$. In order to consider heliostat soiling, a degradation function $\delta_{h}(t)$ for heliostat $h$ at time $t$ must be defined, where the optical efficiency and therefore the energy generated by a heliostat will be reduced over time, unless cleaning operations are performed. The degradation function chosen directly models the effect of soiling on the optical efficiency of a given heliostat and is dependent on local weather conditions. In this study, the degradation function is assumed to be linear in time.

Due to both routing and environmental costs, not all heliostats should be cleaned every day. If the analysis is performed for a single heliostat within the field, which is to be cleaned $r$ times during a cleaning schedule, one can determine the optimal periods to perform cleaning operations in order to maximise optical efficiency of the heliostat. Ignoring routing issues, the solution is simple: the cleaning operations should be done periodically. Indeed, if we denote the efficiency of a fixed heliostat by $f(s)$, where $s$ is the allocated cleaning period, we can define the total efficiency across time as:

$$
F(s):=\int_{0}^{s} f(t) d t
$$

where $F^{\prime} \equiv f(x)$ is decreasing and $F$ is therefore a concave function.

We can find the total efficiency of a heliostat, across a cleaning schedule with multiple cleaning instants, by calculating:

$$
\tilde{F}\left(s_{1}, \ldots, s_{r}\right)=\int_{0}^{s_{1}} f(t) d t+\int_{0}^{s_{2}} f(t) d t+\ldots+\int_{0}^{s_{r}} f(t) d t
$$

Our aim is to maximise the efficiency of the heliostats across the cleaning schedule:

$$
\text { Maximise } \tilde{F}\left(s_{1}, \ldots, s_{r}\right) \text {, }
$$

subject to:

$$
s_{1}, \ldots, s_{r} \geq 0
$$




$$
\sum_{i=1}^{r} s_{i}=T .
$$

Since $F$ is a concave function $\frac{1}{r} \tilde{F}\left(s_{1}, \ldots, s_{r}\right)=\frac{1}{r} \sum_{i=1}^{r} F\left(s_{i}\right) \leq F\left(\frac{T}{r}\right)$ which implies $\tilde{F}\left(s_{1}, \ldots, s_{r}\right) \leq r F\left(\frac{T}{r}\right)=\tilde{F}\left(\frac{T}{r}, \frac{T}{r}, \ldots, \frac{T}{r}\right)$. Therefore, the best strategy is to perform periodic cleaning operations.

While the previous analysis shows that the optimal cleaning strategy for one single heliostat is given by a periodic schedule, the problem to be addressed involves many heliostats.

In the next section, we formulate the clustering procedure as an optimisation problem for the allocation of heliostats to groups.

\subsection{Clustering heliostats}

Addressing the scheduling problem and subsequent routing problems may be unmanageable, since the considered heliostat field may contain thousands of units. For this reason it is worthwhile applying a clustering strategy $[14,16]$ before the optimisation of a cleaning schedule, reducing problem size and complexity.

We look to cluster the heliostats in the field into distinct groups. Groups should not be geographically disperse, since this would increase routing costs. Moreover, since the cleaning schedule will be identical for heliostats in the same group, such heliostats should generate a similar amount of energy. It is considered that the energy $E_{h}$ generated by a heliostat $h$ is the average produced along one day, as shown in Figure 2 for the PS10 SPT plant in Sanlúcar la Mayor, Seville [1].

A dissimilarity function is then introduced: for any pair of heliostats, $h, h^{\prime}$, let $\lambda_{h h^{\prime}}$ denote the dissimilarity between $h$ and $h^{\prime}$, given by $\lambda_{h h^{\prime}}=\alpha \operatorname{Dist}\left(h, h^{\prime}\right)+\beta\left|E_{h}-E_{h^{\prime}}\right|$, where $\alpha, \beta>0$ are given constants and $\operatorname{Dist}\left(h, h^{\prime}\right)$ is the physical distance between the heliostats $h$ and $h^{\prime}$.

The choice of constants $\alpha$ and $\beta$ determines the importance of distance and energy in the clustering optimisation. These values are chosen according to the plant being modelled and the interest of the user in maximising energy or minimising distance. 


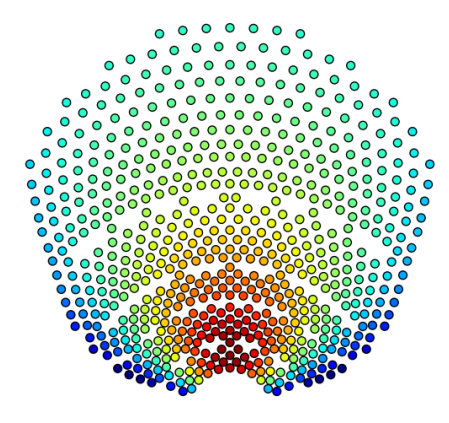

Figure 2: Average reflected radiation per heliostat

This clustering can be expressed as a $p$-median problem, as in $[3,12,18]$. Heliostats can be viewed as clients and $p$ potential plants have to be selected, the primary heliostats, which are the central heliostats of the groups. This is modelled as a binary linear problem in which the overall dissimilarity between each heliostat and its associated primary heliostat is to be minimised. More precisely, let us denote by $H$ the set of heliostats in the SPT field. For any heliostat $h \in H$, let us set

$$
y_{h}= \begin{cases}1, & \text { if } h \text { is a primary heliostat } \\ 0, & \text { otherwise. }\end{cases}
$$

For heliostats $h$ and $h^{\prime} \in H$, let us set

$$
a_{h h^{\prime}}= \begin{cases}1, & \text { if } h^{\prime} \text { is allocated to group with primary heliostat } h \\ 0, & \text { otherwise. }\end{cases}
$$

We then constrain the optimisation problem by requiring that each heliostat $h$ may only be allocated to one group, with primary heliostat $h$ :

$$
\sum_{h \in H} a_{h h^{\prime}}=1 \quad \forall h^{\prime} \in H
$$

We also limit the number of clusters to a constant value $P$ :

$$
\sum_{h \in H} y_{h}=P
$$

Finally, we set a limit, $S$, on the number of heliostats allocated to each group.

$$
\sum_{h^{\prime} \in H} a_{h h^{\prime}} \leq S y_{h} \quad \forall h \in H
$$


Finally, the objective function can be written in the form

$$
\sum_{h^{\prime} \in H} \sum_{h \in H} \lambda_{h h^{\prime}} a_{h h^{\prime}}
$$

which is to be minimised.

\subsection{Schedule Optimisation}

Once heliostats have been clustered into groups, as detailed in Section 2.2, we allocate groups to cleaning periods in order to optimise the overall cleaning schedule. The objective of the optimisation procedure is to maximise the total energy generated over the schedule duration, whilst considering the allocation of heliostat groups to cleaning periods and the subsequent degradation of efficiency of each heliostat.

Using the reflected energy from each heliostat in the field (which is calculated in a preprocessing step) and the assumed degradation function, we maximise the total energy reflected by all heliostats across the cleaning period. In what follows we show how to address the scheduling problem by using Mathematical Optimisation.

We denote by $C$ the set of periods and $\bar{H}$ the set of primary heliostats, obtained by solving the p-median problem described in Section 2.2. Moreover, we define the binary variable $x_{h c}$, where:

$$
x_{h c}= \begin{cases}1, & \text { if group with primary heliostat } h \text { is cleaned in period } c \\ 0, & \text { otherwise. }\end{cases}
$$

for any $h \in \bar{H}$ and $c=1, \ldots, C$.

In order to determine the loss of efficiency for a specific period, it is necessary to also define the binary variable $z_{h c r}$, which accounts for whether a heliostat group has been cleaned in the previous periods with relation to the current period. Thus, for any $h \in \bar{H}, c \in C$ and $r=0, \ldots, c$, we set:

$$
z_{h c r}= \begin{cases}1, & \text { if group with primary heliostat } h \text { was cleaned } r \text { periods before period } c \\ 0, & \text { otherwise }\end{cases}
$$

For instance, if we are considering whether or not to clean heliostat group 3 during a schedule of length 2 , we would include the variables $z_{310}, z_{311}, z_{320}, z_{321}$ and $z_{322}$.

By considering the energy generated by a heliostat with full optical efficiency, subtracting the amount of energy lost per period it is not cleaned, $E_{h c r}$, and summing for all heliostats over all periods, one can calculate the overall energy generated by the field. Using the binary variables $z_{h c r}$, our objective is then

$$
\sum_{h \in \bar{H}} \sum_{c \in C} \sum_{r} E_{h c r} z_{h c r}
$$

to be maximised. 
We constrain this objective function by requiring that each heliostat group must be cleaned at least once across all periods:

$$
\sum_{c \in C} x_{h c} \geq 1 \quad \forall h \in \bar{H} .
$$

This constraint is applied, as it is assumed that all groups of heliostats in the field provide significant energy to the system, and must all be maintained in the long term. In the case where a heliostat field has not been optimised, it may be of interest to not enforce this constraint, and investigate whether or not some heliostats are never cleaned and therefore may not be of significant value to the overall system.

We also assume that each cleaning route may clean at most $\tau$ heliostats:

$$
\sum_{h \in \bar{H}} S_{h} \cdot x_{h c} \leq \tau \quad \forall c \in C,
$$

where $S_{h}$ denotes the cardinality of the group with primary heliostat $h$.

This constraint models the water carrying capacity of the cleaning vehicle, as well as the time taken during a particular cleaning period, and it may be changed according to each particular SPT plant specifications.

We also introduce the following constraints:

$$
z_{h c c} \leq 1-x_{h i} \quad \forall i=1, \ldots, c, \forall c=1, \ldots, C, \forall h \in \bar{H},
$$

where $z_{h c c}$ will be zero if heliostat group with primary heliostat $h$ is cleaned in any period;

$$
z_{h c r} \leq x_{h(c-r)} \quad \forall r=0, \ldots, c-1, \quad \forall c=1, \ldots, C, \forall h \in \bar{H},
$$

where $z_{h c r}$ will be zero if heliostat group with primary heliostat $h$ is not cleaned $r$ periods before period $c$;

$$
z_{h c r} \leq 1-x_{h(c-r+1)} \quad \forall r=1, \ldots, c-1, \forall c=1, \ldots, C, \forall h \in \bar{H},
$$

where $z_{h c r}$ will be zero if heliostat group with primary heliostat $h$ is cleaned in the period $r$, before period $c$.

Finally, we include a constraint to remove the degradation penalty for a heliostat group cleaned in a particular period:

$$
x_{h c}+\sum_{r=1}^{c} z_{h c r}=1 \quad \forall c \in C, \forall h \in \bar{H} .
$$

With the objective function (14) and the constraints (15) - (20), we now have a constrained binary integer linear program for the variables $x_{h c} \in\{0,1\}, z_{h c r} \in\{0,1\}$, with $h \in \bar{H}, c \in C$ and $r=1, \ldots, c$. 


\subsection{Cluster Scheduling. Local Search}

The cleaning schedule found using the method discussed in Section 2.3 produces a set of subsequent routing problems, where the groups assigned to each period form the clients within the local routing problem, as in [5, 27]. When considering each routing problem, the objective is to minimise the route length whilst visiting all groups, however the operational ease of use for the cleaning vehicle should be seen as a factor of importance, as in [20]. In order to reduce operational costs, it may be of benefit to use heuristics to alter the cleaning schedule found, at a cost of total schedule energy.

We consider local search heuristics to produce routing refinement options, which may be used to improve the routes across the schedule, at a cost to the overall energy produced. The initial schedule designed in Section 2.3 can be refined by means of a 2-opt local search technique, by considering the optimisation of two routing problems at once, for two adjacent periods in the schedule, where groups can be swapped between the periods. This technique is then iterated across each pair of periods, and the sequence repeated until no further improvement to the solution is found. Observe that each iteration of the 2-opt local search amounts to solving an optimisation problem. Such an optimisation problem is much smaller in size than an overall optimisation procedure, since only the clusters in two consecutive periods are considered for reallocation. The formulation developed in this section is an adaptation of the Vehicle Routing Problem (VRP), see [17, 19], where we minimise the route of the cleaning vehicle for each period, whilst considering energy loss as a penalty factor.

Let us denote by $P$ the number of groups and set

$$
x_{i j p}= \begin{cases}1, & \text { if group } i \text { is allocated to period } j \text { in route position } p \\ 0, & \text { otherwise, }\end{cases}
$$

for any $i=1, \ldots, P$, any $j=1,2$ and any $p=1, \ldots, P-1$.

Let $l_{h h^{\prime}}$ be the distance between primary heliostats $h$ and $h^{\prime}$, and let $D_{i}$ be the energy difference caused if group $i$ swaps periods. Using the binary variables $x_{i j p}$ and the distances between groups $l_{h h^{\prime}}$, we minimise the overall route length for the two periods considered, whilst adding an energy based penalty $D_{i}$ if a group has swapped period.

We therefore look to address the optimisation problem

$$
\text { Minimise } \sum_{i \neq i^{\prime}} \sum_{p=1}^{P-1} x_{i, j, p} x_{i^{\prime}, j, p+1} \cdot \alpha l_{i, i^{\prime}}+\beta D_{i} \quad \forall j
$$

We constrain this problem by permitting each group to be allocated to one day and in only one position:

$$
\sum_{j=1}^{2} \sum_{p=1}^{P-1} x_{i, j, p}=1 \quad \forall i=1, \ldots, P
$$


We also ensure that each position in the route on each day may only have one group in total:

$$
\sum_{i=1}^{P} x_{i, j, p}=1 \quad \forall j=1,2, \forall p=1, \ldots, P-1 .
$$

And, finally,

$$
\sum_{i=1}^{P} x_{i, j, p} \in\{0,1\} \quad \forall j=1,2, \forall p=1, \ldots, P-1 .
$$

The objective function contains the product of two binary variables, using a Fortet scheme as follows.

Let us set

$$
y_{i i^{\prime} p}^{j}=x_{i, j, p} x_{i^{\prime}, j, p+1} .
$$

The objective function then becomes:

$$
\sum_{i \neq i^{\prime}} \sum_{p=1}^{P-1} y_{i i^{\prime} p}^{j} \cdot \alpha l_{i, i^{\prime}}+\beta D_{i} \quad \forall j
$$

to be minimised.

In the next section, we illustrate the developed optimisation procedure by producing a cleaning strategy for a sample SPT plant and solve the subsequent routing problems.

\section{Results}

\subsection{Problem Description}

The method developed in this work is applied to the PS10 plant in Sanlúcar la Mayor, Seville [1], which has 624 heliostats in a North facing field with a layout as shown in Figure 3. The parameters used are given in Table 1.

First, heliosts are clustered as described in Section 2.2, considering 52 groups of 12 heliostats $(P=52, S=12)$ and the optimisation procedure developed in Section 2.3.

For the purposes of this study, where we are considering discrete time instants, we will assume a linear degradation function. This implies that, for each period that the heliostat is not cleaned, the efficiency decreases by a constant quantity $\delta$. This quantity will vary with the SPT plant location and heliostat construction, and will be assumed to be between $5 \%$ loss per period for the purpose of this study.

The initial cleanliness of the heliostat field is variable, and will have a distinct effect on the result of the optimisation procedure. For the purposes of this study, we will 
assume an initial random efficiency loss between 0 and $10 \%$ for each heliostat in the field.

The program was written using the Python programming language and utilised the Gurobi optimisation package [15] on a computer with specifications: Intel@ Core $^{\mathrm{TM}}$ i7-7700HQ CPU @ $2.80 \mathrm{GHz}$. In the clustering analysis, as detailed in Section 2.2, the $\alpha$ and $\beta$ weighting constants were chosen as 0.6 and 0.4 respectively, causing the distance between heliostats to be slightly more important than their difference in energy generation.

For simplicity, the incident radiation on the heliostat field is assumed to be identical for each period considered, and the maximum possible reflected radiation for each heliostat, shown in Figure 4, is averaged over one period. The data point chosen was midday with clear skies from the data set in [2].

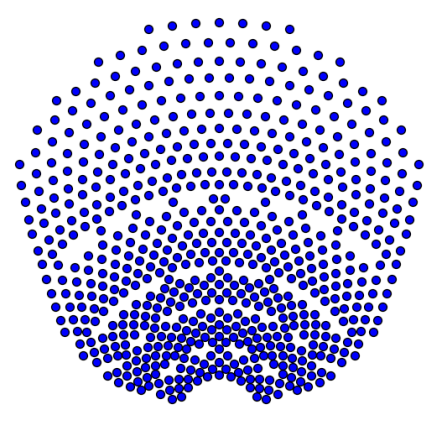

Figure 3: PS10 Heliostat field layout

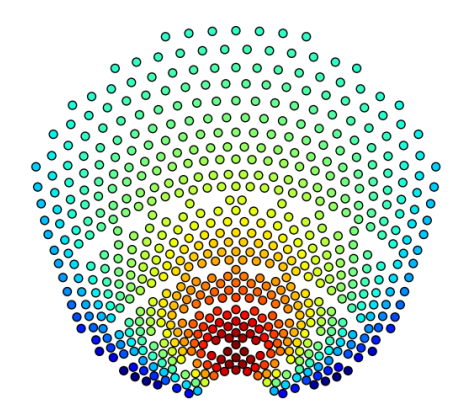

Figure 4: Average reflected radiation

\begin{tabular}{|l|l|l|}
\hline Parameter & Value & Summary \\
\hline$P$ & 52 & Number of groups in clustering optimisation. \\
\hline$S$ & 12 & $\begin{array}{l}\text { Number of heliostats in each group for clustering } \\
\text { optimisation. }\end{array}$ \\
\hline$H$ & 624 & Number of heliostats in field. \\
\hline$\alpha$ & 0.4 & $\begin{array}{l}\text { Importance of distance in clustering optimisa- } \\
\text { tion. }\end{array}$ \\
\hline$\beta$ & 0.6 & Importance of energy in clustering optimisation. \\
\hline$\tau$ & 50 & Maximum number of heliostats in a route. \\
\hline$\delta$ & $5 \%$ & Efficiency degradation per period. \\
\hline$\delta_{0}$ & $0-10 \%$ & Initial heliostat cleanliness. \\
\hline
\end{tabular}

Table 1: Parameter values 


\subsection{Schedule Optimisation}

We first optimise the clustering of heliostats in the field, using the method described in Section 2.2. Figure 5 shows four of the optimised groups, which can be seen as black coloured heliostats. From this image, it can be seen that the groups are relatively compact, and there are few isolated heliostats in the same group. Considering the energy profile from Figure 4, it can be seen that the groups have also been clustered using similar energy profiles, which is an obvious consequence due to the choice of objective function in the p-median problem used for building the clusters.
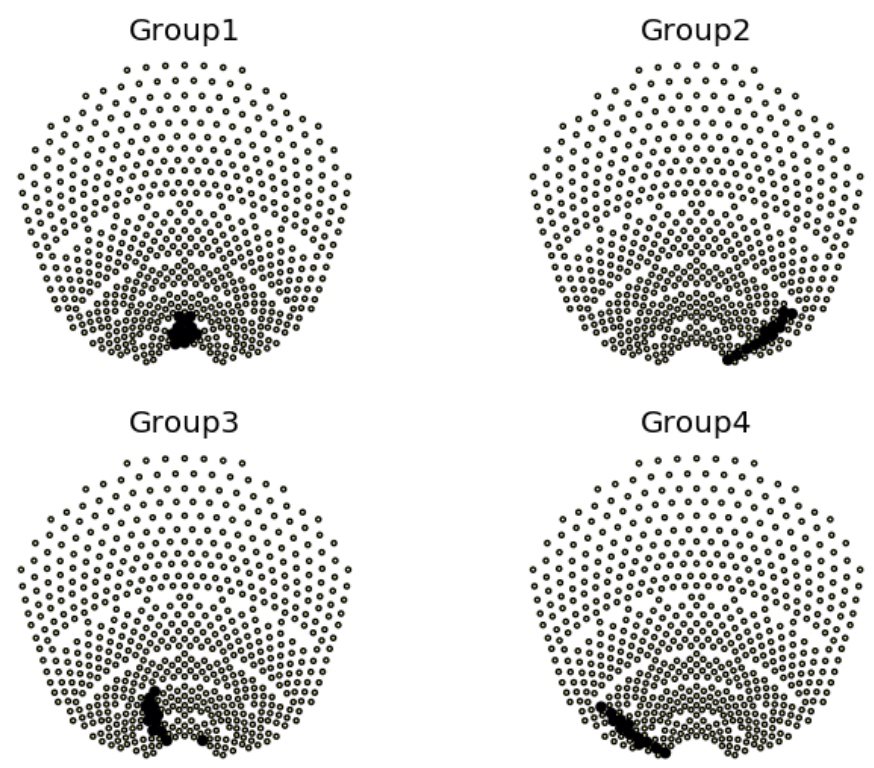

Figure 5: Optimised Heliostat Grouping (groups 1-4)

We then look to optimise the cleaning schedule problem, using the grouping already obtained, considering a schedule of 16 days. Figure 6 shows the computed cleaning schedule, where at each period, the cleaned heliostats are marked with white points. Figure 7 shows the resultant energy production of each heliostat in the field. From these figures, we can see the evolution of energy production over the schedule due to cluster allocation, and check that, as expected, the heliostats in the centre of the field are kept cleaner than the rest, due to their higher energy efficiency. 


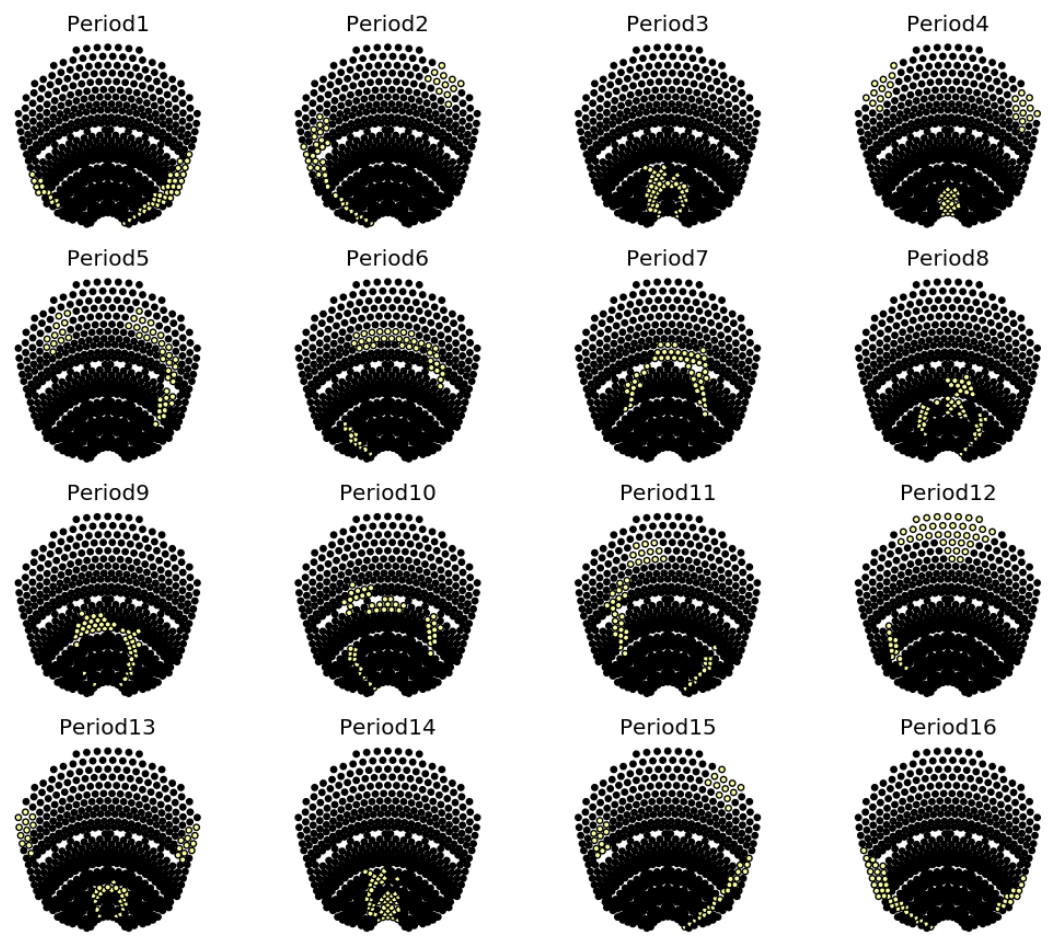

Figure 6: Optimal Cleaning Schedule Allocation 


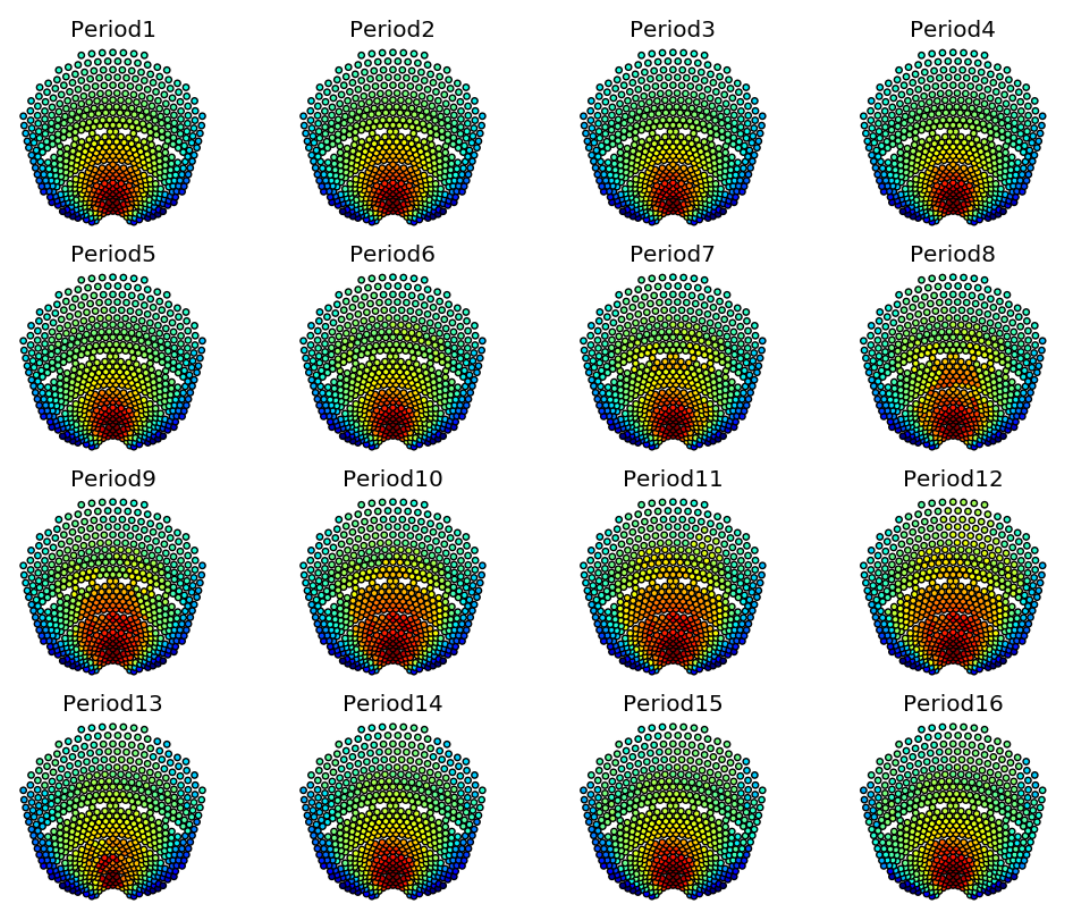

Figure 7: Optimal Cleaning Schedule Energy Profile

From these results, it can be seen that whilst a sub-optimal cleaning schedule with grouping has been found in terms of energy reflected onto the receiver, in certain periods disjoint subtours can be found (for example Period 4 in Figure 6), which are not desired by SPT plant operators and should therefore be removed in a local search phase.

\subsection{Local Search Heuristic}

The cleaning schedule shown in Figure 6 is taken as the initial solution in the swapping algorithm described in Section 2.4. We then obtain the results depicted in Figure 8. This solution was obtained within two iterations through the pairwise rolling optimisation procedure, where an iteration is comprised of $N-1$ pairwise swaps. Comparing Figures 6 and 8 , it can be seen that groups have swapped between periods where the loss in energy was outweighed by the benefit in having less disperse heliostat clusters in the same time periods. 


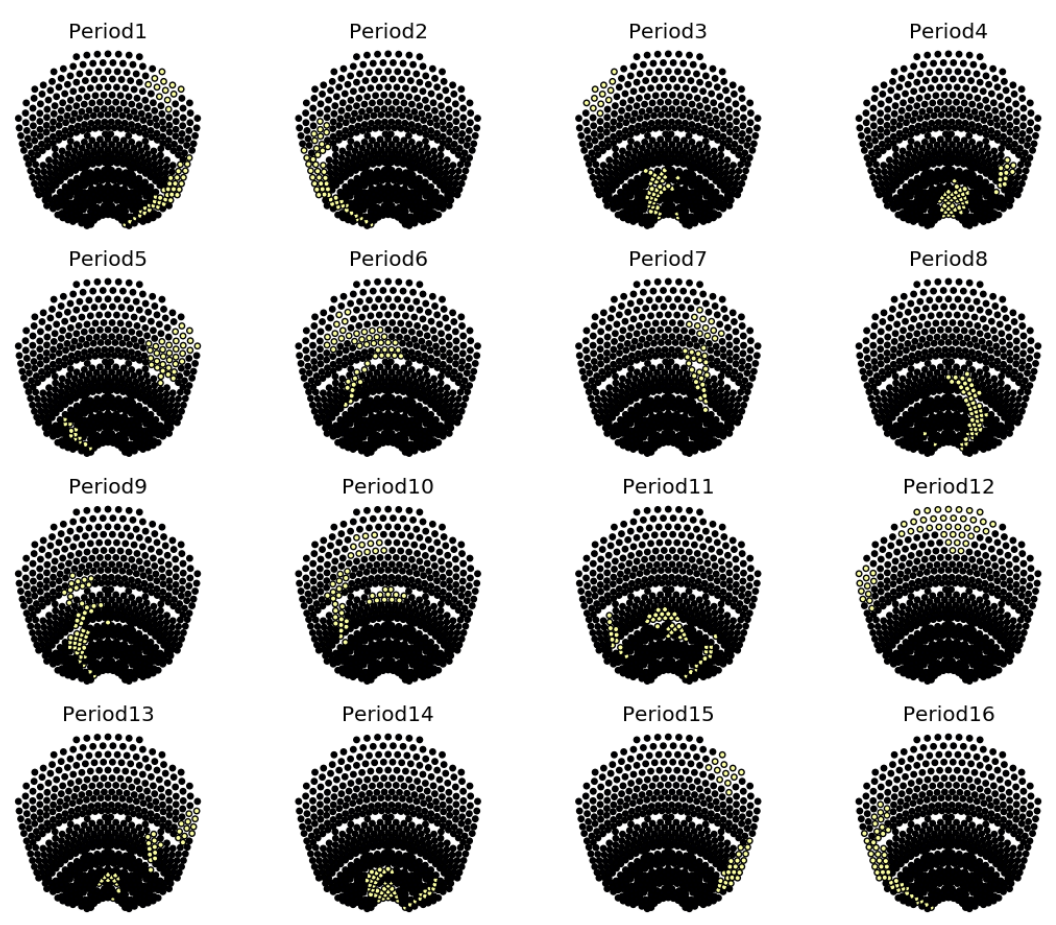

Figure 8: Optimised Cleaning Schedule

With the cleaning schedule obtained in Figure 8, a standard Travelling Salesman Problem (TSP) is then solved for each period in the schedule. For example, Figure 9 shows the optimal route for the allocation of period 2 that can be seen in Figure 8. This route has been found using a Greedy Algorithm implemented in Python. 


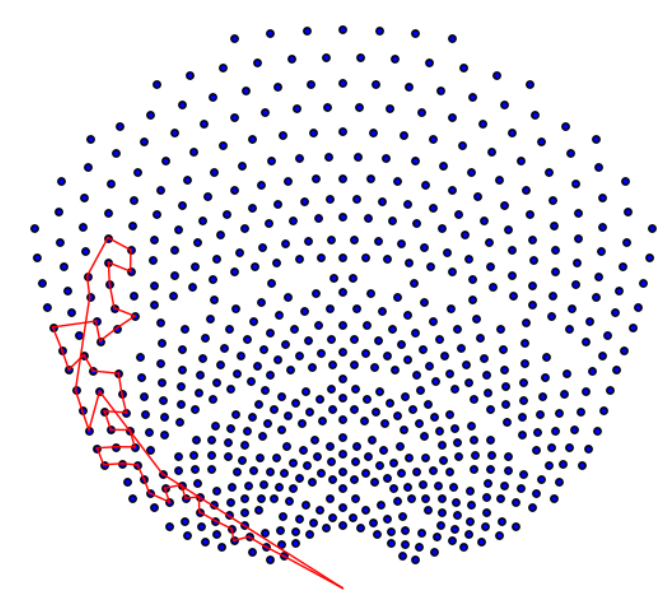

Figure 9: Calculated Route Period 2

The local search heuristic alters the initial solution, yielding better routes for the final solution at the cost of overall collected energy. In this example, the value of total energy collected over the schedule reduces by $2.7 \%$ due to the application of the local search heuristic.

\section{Conclusions}

In this study a procedure has been developed to optimise the cleaning schedule for a SPT plant. This procedure includes a novel heuristic approach to refine the solution, to account for route attractiveness in the final solution. The procedure has been illustrated in a real SPT plant using typical cleaning technology and assumptions on the efficiency degradation of heliostats due to soiling mechanisms. The presented method provides an increase of nearly $5 \%$ in total energy of the schedule, when compared to an assumed cleaning schedule which followed the rows of the heliostats in the plant. Whilst an increase in total energy was found, the real benefit for the method would be found in the design phase of an SPT plant, where the location of heliostats and their access roads could take into account the optimal cleaning schedule.

A $p$-median type linear integer program was developed to perform a clustering analysis, reducing large problem spaces by finding the optimal grouping strategy of the heliostats, considering a weighted objective function of physical distance and energy 
profile between heliostats in the field. The heliostat grouping is then used to obtain an initial cleaning schedule of the heliostat groups over a period of time, in order to maximise the energy reaching the receiver. In our numerical illustration, this program was presented for a schedule of 16 periods, with a route limit of 52 heliostats per period, where the results show an optimal energy profile, but a sub-optimal routing solution for each period.

The initial solution is then refined with a local search heuristic, which pairwise swaps groups of heliostats between consecutive days in the schedule. Each move amounts to solving a linear integer problem, where operational costs are reduced in the routing problems, at a cost of total energy gained during the schedule.

The length of the schedule optimised in Section 3 was 16 periods, which was chosen in order to present a study of an interesting length. This choice will affect the result of the optimisation procedure, due to variable local weather conditions, and further research of interest is to optimise the schedule length chosen.

The example route shown in Figure 9 does not follow the rows formed during the construction of the SPT plant, and assumes that the cleaning vehicle can navigate between heliostats. In the case of the PS10 SPT plant, the application of this route is possible due to spacing between heliostats and the ground topology. However, some SPT plants have obstructions, both natural and man-made, within their fields. These obstructions will limit the paths that the cleaning vehicle can take, and must be taken into account when optimising the cleaning schedule. This can be implemented in the method developed in this study with additional constraints.

The optimisation procedure developed in this work can be utilised by current SPT plant operators optimise their cleaning routes. It can also be utilised in the planning phase of a new SPT plant, where pattern-free heliostat fields will require more complex cleaning routes, which need to be optimised to ensure maximum energy generation.

The application of Operations Research techniques to SPT plants design and operations has many possibilities, where this work could be extended to include; time dependency if cleaning operations are conducted during the day, routing problems with depots due to water carrying capacity of cleaning vehicles, stochastic processes for weather events which have varying effects on the soiling of heliostats, and large scale problems with multiple towers.

This investigation could be used to influence future design of SPT plants, in order to maximise their energy generation and reduce overall costs, by aiding in the design of cleaning schedules whilst adapting the layout of the heliostat field using techniques as in [7]. The results presented use a novel swapping approach for pairs of periods in the schedule. However this is directly extendible for any number of periods at a time.

Another extension to this work could be the investigation into the effects of optimal cleaning schedules, where we look to maximise optical efficiency of the heliostats, against optimal aiming strategies as developed in [2]. 


\section{Acknowledgements}

This research is being supported by the Spanish Government through the research project PCIN-2015-108 and is being conducted within the group MTM2015-65915-R at the University of Seville. 


\section{References}

[1] Abengoa. Abengoa PS10 SPT Plant. [Date Accessed: 22/02/2017], 2017.

[2] Thomas Ashley, Emilio Carrizosa, and Enrique Fernández-Cara. Optimisation of aiming strategies in Solar Power Tower plants. Energy, 2017.

[3] Pasquale Avella, Antonio Sassano, and Igor Vasil. Computational study of largescale p -Median problems. Mathematical Programming, 109:89-114, 2007.

[4] J.G. Barberena, A. Mutuberria Larrayoz, M. Sánchez, and A. Bernardos. State-ofthe-art of Heliostat Field Layout Algorithms and their Comparison. Energy Procedia, 93(March):31-38, 2016.

[5] Sérgio Barreto, Carlos Ferreira, José Paixão, and Beatriz Sousa Santos. Using clustering analysis in a capacitated location-routing problem. European Journal of Operational Research, 179(3):968-977, 2007.

[6] Saeb M. Besarati and D. Yogi Goswami. A computationally efficient method for the design of the heliostat field for solar power tower plant. Renewable Energy, 69(November 2016):226-232, 2014.

[7] E. Carrizosa, C. Domínguez-Bravo, E. Fernández-Cara, and M. Quero. A heuristic method for simultaneous tower and pattern-free field optimization on solar power systems. Computers \& Operations Research, 57:109-122, 2015.

[8] E. Carrizosa, C. Domínguez-Bravo, E. Fernández-Cara, and M. Quero. Optimization of multiple receivers solar power tower systems. Energy, 90:2085-2093, 2015.

[9] E. Carrizosa, Carmen-Ana Domínguez-Bravo, E. Fernández-Cara, and M. Quero. An optimization tool to design the field of a solar power tower plant allowing heliostats of different sizes. International Journal of Energy Research, 2017.

[10] Meriem Chaanaoui, Sébastien Vaudreuil, and Tijani Bounahmidi. Benchmark of Concentrating Solar Power plants : historical, current and future technical and economic development. Procedia - Procedia Computer Science, 83(Seit):782-789, 2016.

[11] N C Cruz, S Salhi, J L Redondo, J D Álvarez, M Berenguel, and P M Ortigosa. Hector, a new methodology for continuous and pattern-free heliostat fi eld optimization. Applied Energy, 225(May):1123-1131, 2018.

[12] Mark S Daskin and Kayse Lee Maass. The p-median problem. Location Science, 2015.

[13] A. Fernández-García, L. Álvarez-Rodrigo, L. Martínez-Arcos, R. Aguiar, and J. M. Márquez-Payés. Study of different cleaning methods for solar reflectors used in CSP plants. Energy Procedia, 49:80-89, 2013. 
[14] M. Grotschel and Y. Wakabayashi. A cutting plane algorithm for a clustering problem. Mathematical Programming, 45:59-96, 1989.

[15] Gurobi. Gurobi Optimization Solver. [Date Accessed: 22/02/2017], 2017.

[16] Pierre Hansen and Brigitte Jaumard. Cluster analysis and mathematical programming. Mathematical Programming, 79(1-3):191-215, 1997.

[17] Maaike Hoogeboom, Maria Battarra, Güneş Erdoğan, and Daniele Vigo. Erratum-Exact Algorithms for the Clustered Vehicle Routing Problem. Operations Research, 64(2):456-457, 2016.

[18] Nenad Mladenović, Jack Brimberg, Pierre Hansen, and José A. Moreno-Pérez. The p-median problem: A survey of metaheuristic approaches. European Journal of Operational Research, 179(3):927-939, 2007.

[19] Caroline Prodhon and Christian Prins. A survey of recent research on locationrouting problems. European Journal of Operational Research, 238(1):1-17, 2014.

[20] Diego Gabriel Rossit, Universidad Nacional, Daniele Vigo, and Fernando Tohm. Improving Visual Attractiveness in Capacitated Vehicle Routing Problems : a Heuristic Algorithm. pages 748-755, 2016.

[21] E.P. Roth and R.B. Pettit. Theeffect of soiling on solar mirrors and techniques used to maintain high relfectivity. This work is supported by the Division of Solar Technology, U.S. Department of Energy (DOE), under contract DE-AC04-76-DP00789. Solar Materials Science, pages 199-227, 1980.

[22] Marcelino Sánchez and Manuel Romero. Methodology for generation of heliostat field layout in central receiver systems based on yearly normalized energy surfaces. Solar Energy, 80(7):861-874, 2006.

[23] Alberto Sánchez-González and Domingo Santana. Solar flux distribution on central receivers: A projection method from analytic function. Renewable Energy, 74:576587,2015 .

[24] Travis Sarver, Ali Al-Qaraghuli, and Lawrence L. Kazmerski. A comprehensive review of the impact of dust on the use of solar energy: History, investigations, results, literature, and mitigation approaches. Renewable and Sustainable Energy Reviews, 22:698-733, 2013.

[25] G. Singh, D. Saini, N. Yadav, R. Sarma, L. Chandra, and R. Shekhar. Dust Deposition Mechanism and Cleaning Strategy for Open Volumetric Air Receiver Based Solar Tower Sub-systems. Energy Procedia, 69:2081-2089, 2015.

[26] Vinayak C. Thalange, Vishwanath H. Dalvi, Sanjay M. Mahajani, Sudhir V. Panse, Jyeshtharaj B. Joshi, and Raosaheb N. Patil. Design, optimization and optical performance study of tripod heliostat for solar power tower plant. Energy, 135:610624, 2017. 
[27] Thibaut Vidal, Maria Battarra, Anand Subramanian, and Güneş Erdolan. Hybrid metaheuristics for the Clustered Vehicle Routing Problem. Computers and Operations Research, 58:87-99, 2015.

[28] Kun Wang, Ya Ling He, Yu Qiu, and Yuwen Zhang. A novel integrated simulation approach couples MCRT and Gebhart methods to simulate solar radiation transfer in a solar power tower system with a cavity receiver. Renewable Energy, 89:93-107, 2016.

[29] Kun Wang, Ya-ling He, Xiao-dai Xue, and Bao-cun Du. Multi-objective optimization of the aiming strategy for the solar power tower with a cavity receiver by using the non-dominated sorting genetic algorithm. Applied Energy, 205(June):399-416, 2017. 